\title{
Estefanofilariose e dermatite nodular ulcerativa em cão: relato de caso
}

\section{Stephanofilariasis and nodular ulcerative dermatitis in dog: case report}

\section{Estefanofilariosis y dermatitis nodular ulcerativa en perros: relato de caso}

\section{Antonio Pereira de Novaes ${ }^{1}$}

Centro Nacional de Pesquisa e Desenvolvimento em Instrumentação Agropecuária da Empresa Brasileira de Pesquisa Agropecuária (CNPDIA). São Carlos, SP, Brasil.

\section{Resumo}

Objetivo: Descrever a ocorrência da estefanofilariose em cão, provocando dermatite nodular ulcerativa na região abdominal. Descrição: Cão mestiço, com aproximadamente dez anos de idade, apresentou dermatite nodular ulcerativa na região abdominal. Suspeitou-se que a etiologia poderiam ser larvas da Stephanofilaria spp. Após remoção cirúrgica da lesão, foi feita a impressão do material em lâmina, fixado com etanol e corado com giensa 1:20 e solução de vermelho congo saturada. A visualização dos parasitas (formas larvais e adultas) foi feita em microscópio óptico (200x). Resultados: O método empregado permitiu a visualização do parasita, confirmando a presença de estefanofilariose no cão. Conclusões: Foi observado que a Stephanofilaria spp também afeta cães. As lesões provocadas por esse parasita não são do tipo comumente encontrado. Não têm cura com a medicação normal e é necessário um tratamento especial baseado em ivermectinas com aplicação tópica ou sistêmica.

Palavras-chave: Filariose, diagnóstico. Dermatite. Úlcera cutânea, quimioterapia. Ivermectina. Cães. 


\section{Introdução}

As filárias são helmintos que têm como vetores normalmente os mosquitos. Estes inoculam as microfilárias no hospedeiro, afetando animais e o próprio homem. Assim, tem-se como exemplo o mal do pântano, que ocorre na Ásia, causando cegueira em pessoas por descolamento mecânico da retina pela Oncocerca. O cão normalmente é afetado pelo gênero Dirofilaria, parasitando o ventrículo direito e artéria pulmonar. Os bovinos, búfalos, ovelhas, elefantes, rinocerontes e cervos são afetados pelo gênero Stephanofilaria (AGRAWAL, SHAH, ${ }^{1}$ 1984). As moscas normalmente são os vetores, que entram em contato com o exsudato de feridas, o qual contém os parasitas e suas formas larvares. Assim, esses helmintos são transportados para outras feridas, desenvolvendo então uma lesão específica, ou seja, dermatite nodular ulcerativa. A lesão é agravada pela ação mecânica desses parasitas, que lesionam os vasos linfáticos, provocando obstrução, inflamação e formação de nódulos, que impedem a cicatrização. Essas lesões podem ocorrer ao redor dos olhos e patas, provocando pododermatites (NOVAES et al., 2,3 1988, 1990; NOVAES,${ }^{4} 2001$ ). É uma doença própria das estações quentes e chuvosas, ocasião nas quais proliferam moscas como a Hematobia irritans e outras. Diversas outras estefanofilárias foram descritas, como a $S$. assamensis, S. zaheeri, S. andamani, S. srivatai (AGRAWAL, SHAH, ${ }^{1}$ 1984), S. stilesi (HIBLER, ${ }^{5}$ 1966; DIES, PRINTCHARD,${ }^{6}$ 1985), S. Kaeli (FADZIL, ${ }^{7}$ 1997) e S. Oknawaensis (CHATTERJEE, ${ }^{8}$ 1983).

No Brasil, as citações desses parasitas foram feitas por Oba et al. ${ }^{9}$ ( 1977), Novaes et al. ${ }^{2,3}$ (1988, 1990) e Novaes ${ }^{4}$ (2001). Os eqüídeos são atingidos pelo gênero Parafilaria (NOVAES, ${ }^{4}$ 2001), que provoca lesões idênticas às já descritas.

\section{Relato do Caso}

O presente caso ocorreu em um cão mestiço, com aproximadamente dez anos de idade, atendido em Clínica Veterinária da cidade de São Carlos, SP, em março de 2005, que apresentou dermatite nodular ulcerativa na região abdominal (Figura 1). Pelas características da lesão, suspeitou-se que a etiologia poderia ser causada por larvas da Stephanofilaria spp.

O nódulo foi removido cirurgicamente (Figura 2) e em seguida feitas impressões do exsudado seroso superficial em lâmina. Após a secção longitudinal, foi realizada impressão em lâmina, do conteúdo do interior da lesão. Após a secagem ao ar livre e a fixação com metanol sobre as lâminas, estas foram coradas por imersão em solução de giensa 1:20 por 1 minuto e em solução saturada de vermelho congo por 1 minuto.

\section{Discussão e Conclusões}

A visualização dos parasitas foi feita por meio de microscópio com aumento de 200 vezes. Eles aparecem em forma de $\mathrm{C}$, conforme a descrição de Hibler $^{5}$ (1966) e Chatterjee ${ }^{8}$ (1983), para o primeiro estágio da Stephanofilaria spp (Figura 3); e também sob a forma de filamento (Figura 4), comprovando a suspeita de que a etiologia da lesão seria a Stephanofilaria spp.

Comprovou-se que a Stephanofilaria spp pode também afetar os cães. As feridas provocadas por esses parasitas não são comuns e não cedem à medicação tradicional. Para o tratamento, devem ser indicadas, prioritariamente, as endectocidas e as ivermectinas, aplicadas tanto na forma tópica como na sistêmica, de acordo com Gill ${ }^{10}$ (1991) e Novaes $^{4}(2001)$. 
NOVAES, A. P. Estefanofilariose e dermatite nodular ulcerativa em cão. Stephanofilariasis and nodular ulcerative dermatitis in dog. Estefanofilariose y dermatitis nodular ulcerativa en perro. Rev. Educ. Contin. CRMV-SP/Contin. Educ. J. CRMV-SP, São Paulo, v. 8, n. 2, p. 97-97, 2005.

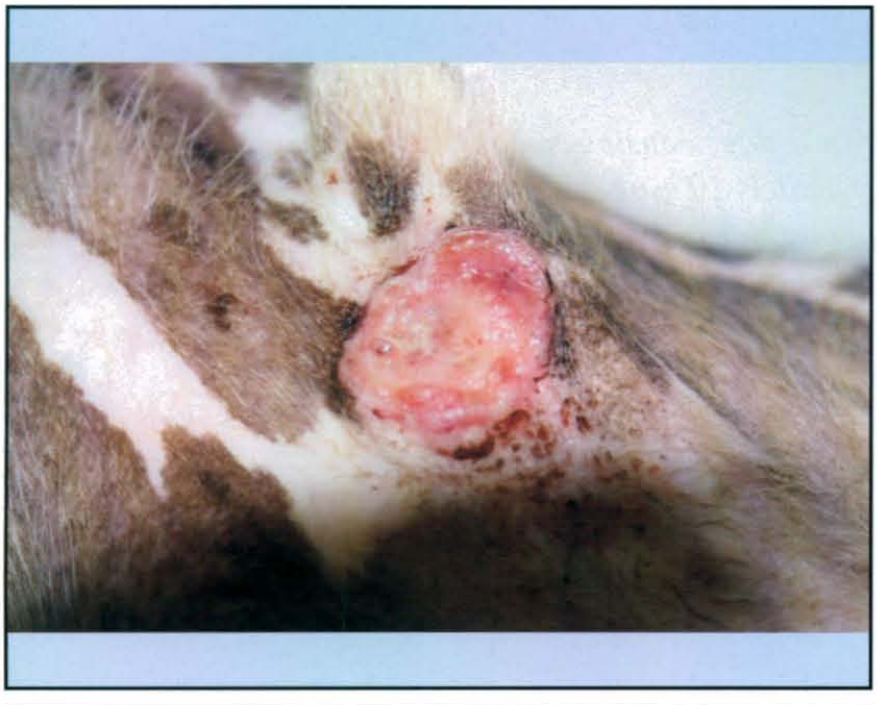

Figura 1 - Fotografia de lesão nodular ulcerativa na região abdominal em cão mestiço com dez anos de idade. São Carlos, SP, 2005

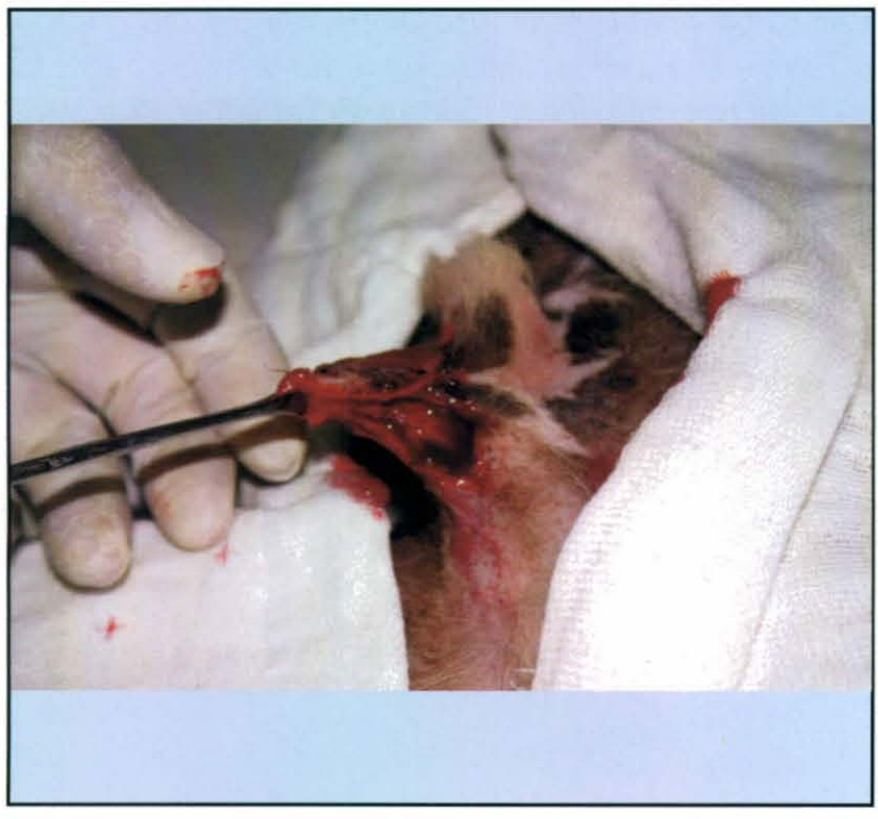

Figura 2 - Fotografia após remoção cirúrgica da lesão nodular ulcerativa de cão mestiço com dez anos de idade. São Carlos, SP, 2005

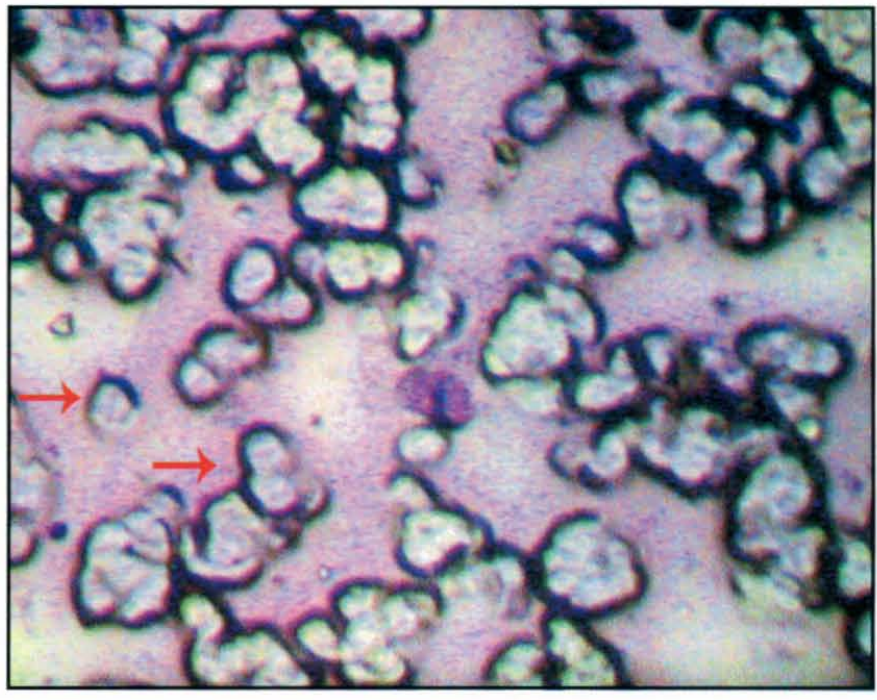

Figura 3 - Fotomicrografia de formas larvais da Stephanofilaria spp. obtidas com máquina eletrônica em microscópio da CNPDIA - EMBRAPA. São Carlos, SP, 2005

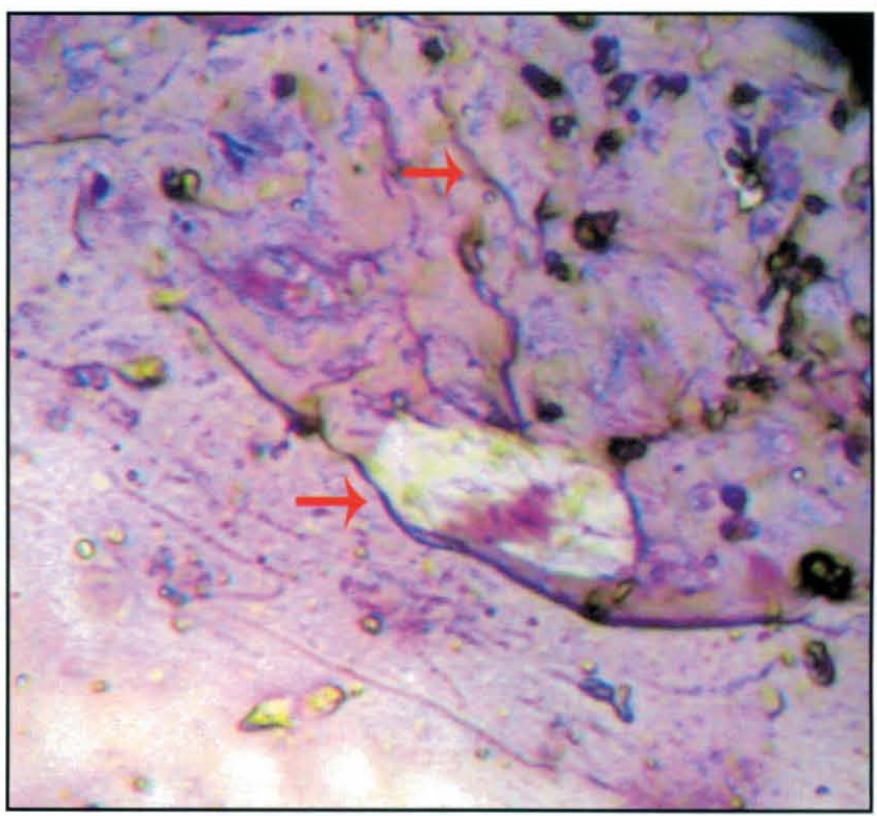

Figura 4 - Fotomicrografia de formas filamentosas da Stephanofilaria spp. obtidas com máquina eletrônica em microscópio da CNPDIA - EMBRAPA. São Carlos, SP, 2005 


\section{Abstract}

Objective: To report on the occurrence of stephanofilariasis in a dog, causing nodular ulcerative dermatitis in the abdominal area. Description: Dog patient, mixed breed, approximately 10 years old, presenting with nodular ulcerative dermatitis in the abdominal area. Suspected etiology was Stephanofilaria spp. After surgical excision of the lesion, an imprint was performed, followed by fixation with ethanol and staining with 1:20 Giensa and saturated Congo red solution. The parasites (larval and adult forms) were identified under optical microscope $(200 x)$. Results: The method employed allowed for visualization of the parasite, thus confirming the occurrence of stephanofilariasis in dogs. Conclusions: It was demonstrated that Stephanofilaria spp can also affect dogs. The lesions caused by this parasite are not of a common type. They do not respond to normal medication and a special treatment based on topical or systemic ivermectin is required.

Key words: Filariasis, diagnosis. Dermatitis. Skin ulcer, drug therapy. Ivermectin. Dogs.

\section{Resumen}

Objetivo: En este trabajo se describe la ocurrencia de la Stephanofilaria en perros, provocando dermatitis nodular ulcerativa en la región abdominal. Descripción: Un perro mestizo con aproximadamente 10 años de edad, mostró dermatitis nodular ulcerativa en la región abdominal. Se sospechó que la etiología podrían ser larvas de Stephanofilaria spp. Después de retirar quirúrgicamente la lesión en la región abdominal, se realizó impresión del material en láminas, fijado con etanol y coloreado con solución de giensa 1:20, además de solución saturada de rojo congo. La visualización del parásito (larvas y formas adultas) se realizó en microscopio óptico (200 x). Resultados: El método utilizado permitió observar claramente los parásitos, confirmando así la presencia de la Stephanofilaria en perros. Conclusiones: Se comprobó que la Stephanofilaria spp puede también afectar a perros. Las heridas provocadas por estos parásitos no son del tipo más común y no se curan con la medicación tradicional, debiendo el tratamiento ser prioritariamente con ivermectinas, aplicadas tanto en la forma tópica como sistémica.

Palabras-clave: Filariosis, diagnóstico. Dermatitis. Úlcera cutánea, quimioterapia. Ivermectina. Perros.

\section{Referências}

1. AGRAWAL, M. C.; SHAH, H.L. Stephanofilarial dermatitis in India. Veterinary Research Communications, v. 8, p. 93-102, 1984.

2. NOVAES, A. P. et al. Dermatite ulcerosa em bovinos provocada por Stephanofilaria. Pesquisa Agropecuária Brasileira, v. 23, p. 927-929, 1988.
3. NOVAES, A. P.; SENA, M. C. O.; MOREIRA, D. P. Stephanofilaria $\mathrm{sp}$ associada a casos de podermatite em bovinos leiteiros. Arquivos de Biologia e Tecnologia, v. 33, p. 575-579, 1990.

4. NOVAES, A. P. Filariose nos animais domésticos. Veterinary News, Rio de Janeiro, v. 52, p. 6-8, 2001. 
NOVAES, A. P. Estefanofilariose e dermatite nodular ulcerativa em cão. Stephanofilariasis and nodular ulcerative dermatitis in dog. Estefanofilariose y dermatitis nodular ulcerativa en perro. Rev. Educ. Contin. CRMV-SP/Contin. Educ. J. CRMV-SP, São Paulo, v. 8, n. 2, p. 97-97, 2005.

5. HIBLER, C. P. Developmemt of Stephanofilaria stilesiu in the horn fly. Journal of Parasitology, v. 52, p. 890-898, 1966.

6. DIES, K. H.; PRINTCHARD, J. Bovine stephanofilarial dermatitis in Alberta. Canadian Veterinary Journal, v. 26, p. 361-362, 1985.

7. FADZIL, N. Infección por Stephanofilaria kaeli en bovinos de la península de Malasia: incidencia y tratamiento. Noticias Médico-veterinárias, v. 1, p. 44-52, 1997.

8. CHATTERJEE, A. A. Description of the microfilaria of Stephanofilaria sp. producing "leg-sore" in cattle in West Bengal (India). Indian Journal of Animal Health, v. 22, p. 163-165, 1983.

9. OBA, M. S. P. et al. Stephanofilaria em bovinos do município de São Carlos, Estado de São Paulo. In: CONFERÊNCIA ANUAL DA SOCIEDADE PAULISTA DE MEDICINA VETERINÁRIA, 32., Pirassununga, SP, 1977. Anais... [S. L.]: Centro Intraunidade de Zootecnia e Indústrias Pecuárias "Fernando Costa" - CIZIP, 1977.

10. GILL, B. S. et al. Treatment of stephanofilariosis (earsore) with ivermectin. Veterinary Parasitology, v. 40, p. 159-163, 1991. 